\title{
Índice de qualidade da dieta de adolescentes residentes no distrito do Butantã, município de São Paulo, Brasil ${ }^{1}$
}

\author{
Healthy eating index of adolescents living in Butanta's \\ district, São Paulo, Brazil
}

Fernanda de Ciccio GODOY2

Samantha Caesar de ANDRADE

Juliana Masami MORIMOTO²

Luana CARANDINA ${ }^{3}$

Moisés GOLDBAUM ${ }^{4}$

Marilisa Berti de Azevedo BARROS 5

Chester Luiz Galvão CESAR²

Regina Mara FISBERG ${ }^{2}$

RES U M O

\section{Objetivo}

Avaliar o índice de qualidade da dieta de adolescentes residentes no Distrito do Butantã do município de São Paulo, SP.

\section{Métodos}

Estudo transversal de base populacional com uma amostra de 437 adolescentes, de ambos os sexos, com idade entre 12 e 19 anos. Foram obtidas amostras probabilísticas em dois estágios, setor censitário e domicílio, da área estudada. O consumo alimentar foi medido pelo método recordatório de 24 horas e a qualidade da dieta avaliada pelo Índice de Qualidade da Dieta adaptado para a realidade local.

\section{Resultados}

Dos adolescentes avaliados, $4 \%$ apresentaram dieta saudável, $68 \%$ dieta que necessita de melhora e $28 \%$ dieta inadequada. O sexo masculino apresentou maior pontuação para os componentes do Índice de Qualidade

\footnotetext{
1 Artigo elaborado a partir dos projetos "Inquérito alimentar em regiões do Estado de São Paulo" (Conselho Nacional de Desenvolvimento Científico e Tecnológico - CNPq - Processo n502948/2003-5) e "Inquérito de Saúde do Estado de São Paulo" (Fundação de Amparo à Pesquisa do Estado de São Paulo - FAPESP - Processo nº8/1499-7).

2 Departamento de Nutrição e Epidemiologia, Faculdade de Saúde Pública, Universidade de São Paulo. Av. Dr. Arnaldo, 725, 01204-906, São Paulo, SP, Brasil. Correspondência para/Correspondence to: R.M. FISBERG. E-mail: <rfisberg@usp.br>.

3 Departamento de Saúde Pública, Faculdade de Medicina, Universidade Estadual Paulista. Botucatu, SP, Brasil.

${ }^{4}$ Departamento de Medicina Preventiva, Faculdade de Medicina, Universidade de São Paulo. São Paulo, SP, Brasil.

5 Departamento de Medicina Preventiva e Social, Faculdade de Ciências Médicas, Universidade Estadual de Campinas. Campinas, SP, Brasil.
} 
da Dieta cereais, hortaliças, leguminosas e variedade da dieta. O aumento no número de anos de estudo do chefe da família apresentou-se associado ao maior consumo dos grupos de alimentos: cereais, verduras e legumes, leite e produtos derivados e variedade de alimentos; a relação foi inversa para o grupo de leguminosas e sódio.

\section{Conclusão}

A maioria dos adolescentes estudada não segue as recomendações dietéticas preconizadas, fato que pode comprometer a saúde futura desses indivíduos.

Termos de indexação: comportamento alimentar; consumo de alimentos; índice de qualidade da dieta; nutrição do adolescente.

\section{A B S T R A C T}

\section{Objective}

To evaluate the Healthy Eating Index among adolescents living in the Butantã district of the city of São Paulo, Brazil.

\section{Methods}

This was a cross-sectional population-based study using a sample of 437 adolescents of both genders, aged between 12 and 19 years. Probabilistic cluster samples were obtained from the study area in two stages (census tracts and homes). Dietary intake was measured by the 24-hour recall method and dietary quality was assessed by means of the Healthy Eating Index, adapted to local realities.

\section{Results}

Among the adolescents assessed, 4\% presented a healthy diet, 68\% a diet that needed some improvement and $28 \%$ an inadequate diet. The scoring for the Healthy Eating Index components of grains, vegetables, pulses and food variety were high among men. Greater schooling levels for heads of families presented an association with greater consumption of grains, vegetables, milk and dairy products, and greater food variety. There was an inverse relationship for pulses and sodium.

\section{Conclusion}

The majority of the adolescents studied did not follow the recognized dietary recommendations, which may compromise these individuals' future health.

Indexing terms: feeding behavior; food consuption; healthy eating index; food intake; adolescents nutrition.

\section{N T R O D U Ç Ã O}

No Brasil, os adolescentes representam $21 \%$ da população, num total de mais de 35 milhões de habitantes, segundo dados do Instituto Brasileiro de Geografia e Estatística do ano de 2000 '. Para a Organização Mundial de Saúde, o limite cronológico da adolescência é a idade entre 10 e 19 anos e 11 meses² $^{2}$

A adolescência é o período de transição entre a infância e a vida adulta, caracterizado pelas intensas mudanças corporais da puberdade e pelos impulsos do desenvolvimento emocional, mental e social. Todas essas mudanças são partes de um processo contínuo e dinâmico, que se inicia na vida fetal, vai se modificando durante a infância sob as influências favoráveis ou desfavoráveis do meio ambiente e do contexto social, e terminam com o completo crescimento físico e a maturação sexual, a consolidação da personalidade, a independência econômica e a integração do indivíduo em seu grupo social².

Devido a essas rápidas modificações, esse novo estágio requer um amplo fornecimento de energia e nutrientes. Durante esse período podem aparecer novos padrões e hábitos alimentares gerados por motivos psicológicos, sociais e socioeconômicos, inclusive influência de amigos, busca de autonomia e identidade, aumento do poder de compra, hábito de preparar rotineira- 
mente seu próprio alimento, a urbanização e o hábito de comer fora de casa. Todas essas alterações podem repercutir, em longo prazo, na saúde futura do indivíduo adulto e na escolha posterior dos alimentos ${ }^{3}$.

Os adolescentes são vulneráveis do ponto de vista nutricional, pois tendem a omitir refeição, especialmente o café da manhã, consumir mais alimentos entre as principais refeições, aumentando a ingestão de açúcares e gorduras saturadas e diminuindo a de micronutrientes. Adolescentes do sexo feminino, insatisfeitas com a imagem corporal, freqüentemente engajam dietas com restrição energética. Esses hábitos alimentares adquiridos na adolescência podem submeter a risco nutricional, inclusive na idade adulta ${ }^{4}$.

Dessa forma, observa-se que a dieta pode influenciar o estado de saúde dos adolescentes e, por isso, tem-se procurado desenvolver instrumentos que avaliem a ingestão global de alimentos e nutrientes, e que incluem vários aspectos da ingestão dietética simultaneamente ${ }^{5}$.

Os índices de qualidade da dieta têm sido desenvolvidos para obter uma medida resumo das principais características da dieta, facilitando a avaliação da qualidade desta em populações ou grupo de indivíduos. Esses têm sido propostos e descritos na literatura científica ${ }^{6}$. O Healthy Eating Index (HEl) foi desenvolvido pelo United States Department of Agriculture (USDA) e considerado, pela American Dietetic Association, adequado para medir a qualidade global da alimentação da população americana, sendo recentemente validado por marcadores bioquímicos ${ }^{7-9}$.

As informações sobre a qualidade da dieta de adolescentes têm recebido atenção crescente, principalmente aquelas com elevado teor de lipídios, energia e carboidratos. Segundo Dietz ${ }^{3}$, a dieta dos adolescentes caracteriza-se pela preferência de alimentos com elevado teor de gordura saturada e colesterol, além da excessiva quantidade de açúcar e sal. Feskanich et al. ${ }^{10}$ descrevem a importância de avaliar a qualidade da dieta de adolescentes, associando o elevado consumo de gordura saturada e sódio com hipertensão e risco de desenvolvimento de doenças cardiovasculares, o aumento na ingestão de açúcares contribuindo para a obesidade e a ocorrência de diabetes tipo 2, e a baixa ingestão de fibras associada a diversos tipos de câncer. Assim, destaca-se a relevância de estudos para avaliação da qualidade da dieta e sua relação com fatores que podem influenciar os hábitos alimentares desse grupo.

Nesse sentido, esse trabalho teve como objetivo avaliar a qualidade da dieta de adolescentes segundo sexo e escolaridade do chefe da família, residentes no Distrito do Butantã do município de São Paulo, SP.

\section{MÉ TO D O S}

Trata-se de um estudo transversal, por meio de inquérito domiciliar, de base populacional. Foi utilizada uma amostragem estratificada, por conglomerados, em dois estágios. Os setores censitários foram agrupados em três estratos segundo o percentual de chefes de família com escolaridade de nível universitário. No primeiro estágio da amostragem foram sorteados, com probabilidade proporcional ao tamanho, 10 setores censitários de cada um dos três estratos, portanto, 30 setores. Após a listagem, em campo, das residências dos setores sorteados, no segundo estágio de amostragem foram sorteados domicílios de cada setor.

O sorteio foi feito de forma a obter um mínimo de 200 entrevistas para cada um dos sexos e idade: 12 a 19 anos do sexo masculino e 12 a 19 anos do sexo feminino. O tamanho mínimo de 200 possibilita estimar uma prevalência de 0,5 com erro de 0,07, considerando um alfa de 0,05 e um efeito de delineamento de 2 . Considerando $20 \%$ de possíveis perdas, foram sorteados 250 indivíduos para cada domínio.

A população de estudo foi composta por 437 adolescentes, de ambos os sexos, com idade entre 12 e 19 anos, residentes no Distrito do Butantã, município de São Paulo, SP. Foram 
excluídos os indivíduos que consumiram menos de $500 \mathrm{kcal}$ e mais de $4000 \mathrm{kcal}(n=9)$.

Os dados foram coletados no período de abril de 2001 a setembro de 2002, por meio de questionário aplicado em visita domiciliar por entrevistadores previamente treinados, sendo respondidos diretamente pelos adolescentes sorteados. Neste estudo foi utilizada a informação referente às características demográficas (sexo, idade, escolaridade do chefe da família) e de estilo de vida (consumo alimentar). O consumo alimentar foi medido a partir da aplicação do Método Recordatório de 24 horas (R24h) nos indivíduos selecionados com representação de todos os dias da semana.

Anteriormente à digitação dos dados de consumo alimentar foi realizada a crítica dos R24h, com o objetivo de identificar as principais e importantes falhas do entrevistador na obtenção da informação. O cálculo do valor nutritivo dos alimentos consumidos foi realizado utilizando-se o programa Virtual Nutri (versão 1.0 for Windows, 1996) com banco de dados de alimentos modificado. No banco de dados de alimentos do programa foram introduzidas as informações da composição química dos alimentos da USDA ${ }^{11}$, Philippi ${ }^{12}$ e Souci et al. ${ }^{13}$. A composição dos alimentos industrializados foi obtida a partir das informações nutricionais disponíveis nos rótulos dos alimentos industrializados e/ou serviços de atendimento ao consumidor (SAC). As preparações caseiras foram desmembradas em seus respectivos ingredientes, a fim de melhor classificar os alimentos segundo os grupos da pirâmide alimentar. Para tal, foram utilizadas as padronizações de receitas propostas por Pinheiro et al. ${ }^{14}$ e Fisberg \& Villar ${ }^{15}$.

Para avaliação da qualidade da dieta foi utilizado o Índice de Qualidade da Dieta (IQD), proposto por Kennedy et al. ${ }^{7}$ e adaptado por Fisberg et al. ${ }^{16}$ para a realidade local. Este índice é obtido por uma pontuação distribuída em dez componentes que caracterizam diferentes aspectos de uma dieta saudável. Cada componente é avaliado e pontuado de zero a dez, sendo que os valores intermediários foram calculados na proporção em que são consumidos. O IQD proposto por Fisberg et al. ${ }^{16}$ foi alterado para utilização neste estudo: o componente "gordura saturada" foi substituído pelo "grupo das leguminosas" e considerou-se o tamanho da porção de cada alimento, para o cálculo da variedade da dieta. A opção pela separação das leguminosas justificou-se pelo hábito alimentar do brasileiro de consumir o feijão, e sua inclusão no mesmo grupo das carnes e ovos poderia levar à superestimação da ingestão desse grupo.

Dessa forma, neste índice os seis primeiros componentes são representados pelos grupos de alimentos, três componentes são representados pelos nutrientes: gordura total, colesterol e sódio, e o último, pela variedade da dieta.

O Quadro 1 resume os componentes do IQD e os critérios definidos para a atribuição das pontuações máxima e mínima.

O processamento de dados foi realizado com o auxílio de um programa desenvolvido no software Statiscal Package for the Social Sciences (SPSS, versão 10, 1999, SPSS Inc., Chicago, Illinois, EUA) específico para este estudo, o qual calcula e atribui a pontuação para cada componente do IQD e para cada indivíduo. Os indivíduos foram classificados em três categorias definidas pela distribuição dos escores de qualidade da dieta encontrados na população alvo. O escore total dos indivíduos foi dividido em três categorias: abaixo de 51 pontos - dieta "inadequada"; entre 51 e 80 pontos - dieta que "necessita de modificação"; e superior a 80 pontos - dieta "saudá-

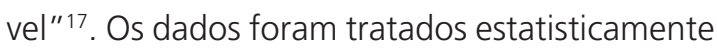
utilizando-se os softwares Epi Info (versão 6.04), SPSS (versão 10) e STATA (versão 7). Inicialmente os dados foram ponderados em função da real distribuição de estratos socioeconômicos na população. Foram calculados a média e o erro padrão. O teste " $t$ " de Wald foi utilizado para verificar a existência de diferenças estatísticas entre as médias do IQD entre categorias das variáveis de estudo, como sexo e escolaridade do chefe da família. 
O projeto foi aprovado pelo Comitê de Ética da Faculdade de Saúde Pública da Universidade de São Paulo (USP).

\section{RESULTADOS}

A média de idade dos adolescentes foi 15,5 anos (Erro-padrão - EP=0,17). A proporção de adolescentes do sexo masculino foi semelhante à do sexo feminino. Quanto à escolaridade do chefe da família, um terço dos indivíduos apresentou entre quatro a sete anos de estudo.

A média de pontos do IQD foi de 58,42 $(E P=0,83)$, sendo que $96 \%$ dos adolescentes avaliados apresentaram dieta inadequada ou que necessita de modificações. Os adolescentes do sexo masculino apresentaram média de pontos do IQD superior ao sexo feminino. A média de pontos do IQD aumentou segundo a escolaridade do chefe da família, porém não houve diferença estatisticamente significante (Tabela 1).

As médias para a pontuação dos componentes do IQD apresentaram-se baixas $(<5)$ para hortaliças, frutas e leite e derivados; e elevadas ( $>7$ ) para cereais, pães e raízes; carnes e ovos; e colesterol, independentemente do sexo. Entretanto, os adolescentes do sexo masculino apresentaram maior pontuação dos seguintes componentes: cereais, pães e raízes, hortaliças; leguminosas, e variedade da dieta, enquanto que para sódio ocorreu o inverso (Tabela 2).

Em relação à escolaridade do chefe da família, as médias de pontuação dos componentes do IQD foram elevadas para cereais, pães e raízes; carnes e ovos, e colesterol; e baixas para hortaliças e frutas de forma independente. Contudo, para os componentes cereais, pães e raízes; hortaliças,

Quadro 1. Descrição, variação da pontuação e critérios para pontuação máxima e mínima de cada componente do Índice de qualidade da dieta.

\begin{tabular}{|c|c|c|}
\hline Componente & $\begin{array}{l}\text { Critério para a pontuação mínima } \\
\text { (0 ponto })\end{array}$ & $\begin{array}{l}\text { Critério para a pontuação máxima } \\
\text { (10 pontos) }\end{array}$ \\
\hline 1. Grupo dos cereais, pães, tubérculos e raízes & Sem consumo & 5 a 9 porções \\
\hline 2. Grupo das hortaliças & Sem consumo & 4 a 5 porções \\
\hline 3. Grupo das frutas & Sem consumo & 3 a 5 porções \\
\hline 4. Grupo do leite e derivados & Sem consumo & 3 porções \\
\hline 5. Grupo das carnes e ovos & Sem consumo & 1 a 2 porções \\
\hline 6. Grupos das leguminosas & Sem consumo & 1 porção \\
\hline 7. Gordura total & $45 \%$ ou mais do VET & $\leq 30 \%$ e $>20 \%$ do VET** \\
\hline 8. Colesterol & $450 \mathrm{mg}$ ou mais & $300 \mathrm{mg}$ ou menos \\
\hline 9. Sódio & $4800 \mathrm{mg}$ ou mais & $2400 \mathrm{mg}$ ou menos \\
\hline 10. Variedade da dieta & $\begin{array}{l}3 \text { ou menos diferentes tipos de } \\
\text { alimentos ao dia* }\end{array}$ & $\begin{array}{l}8 \text { ou mais diferentes tipos de alimentos } \\
\text { ao dia* }\end{array}$ \\
\hline
\end{tabular}

* Alimentos que contribuam com, pelo menos, metade da porção do grupo alimentar correspondente; **avaliando os valores obtidos em relação ao percentual de gordura, não houve valor inferior a $20 \%$.

Tabela 1. Pontos do índice de qualidade da dieta (IQD) segundo sexo e escolaridade do chefe da família. São Paulo, 2004.

\begin{tabular}{lccccc}
\hline Variável & Categoria & $n$ & Média IQD & Erro-padrão & $P^{* *}$ \\
\hline \multirow{2}{*}{ Sexo } & Masculino & 222 & 59,89 & 0,84 & 0,01 \\
\hline Escolaridade do chefe da & Feminino & 215 & 56,95 & 1,05 & 2,30 \\
família (anos)* & 0 a 3 & 105 & 55,62 & 1,03 & 1,26 \\
& 4 a 7 & 151 & 58,13 & 59,47 & 1,97 \\
\hline
\end{tabular}

* Cinco indivíduos sem informação sobre escolaridade do chefe da família; ** Teste " $t$ " de Wald. 
Tabela 2. Média de pontos dos componentes do índice de qualidade da dieta (IQD) por sexo. São Paulo, SP, 2004.

\begin{tabular}{|c|c|c|c|c|c|}
\hline \multirow{3}{*}{ Componentes do IQD } & \multicolumn{4}{|c|}{ Sexo } & \multirow{3}{*}{$p^{* *}$} \\
\hline & \multicolumn{2}{|c|}{ Masculino } & \multicolumn{2}{|c|}{ Feminino } & \\
\hline & Média & $E P^{*}$ & Média & EP & \\
\hline Cereais, pães, raízes & 8,28 & 0,17 & 7,18 & 0,20 & $<0,01$ \\
\hline Hortaliças & 4,63 & 0,32 & 3,62 & 0,26 & 0,01 \\
\hline Frutas & 2,60 & 0,30 & 2,88 & 0,41 & 0,61 \\
\hline Leite e derivados & 3,86 & 0,49 & 3,78 & 0,26 & 0,88 \\
\hline Carnes e ovos & 9,01 & 0,20 & 8,58 & 0,23 & 0,20 \\
\hline Leguminosas & 5,93 & 0,58 & 4,63 & 0,34 & 0,03 \\
\hline Gordura total & 5,79 & 0,18 & 5,48 & 0,20 & 0,21 \\
\hline Colesterol & 7,92 & 0,35 & 8,36 & 0,25 & 0,20 \\
\hline Sódio & 4,58 & 0,37 & 6,36 & 0,38 & $<0,01$ \\
\hline Variedade da dieta & 7,29 & 0,20 & 6,07 & 0,25 & $<0,01$ \\
\hline
\end{tabular}

*EP: erro-padrão; **Teste " $t$ " de Wald.

Tabela 3. Média de pontos dos componentes do índice de qualidade da dieta (IQD) por escolaridade do chefe da família (anos). São Paulo, SP, 2004.

\begin{tabular}{|c|c|c|c|c|c|c|c|c|c|}
\hline \multirow{3}{*}{ Componentes do IQD } & \multicolumn{8}{|c|}{ Escolaridade do chefe da família (anos) } & \multirow{3}{*}{$p^{* *}$} \\
\hline & \multicolumn{2}{|c|}{$0-3$} & \multicolumn{2}{|c|}{$4-7$} & \multicolumn{2}{|c|}{$8-11$} & \multicolumn{2}{|c|}{12 ou +} & \\
\hline & Média & EP & Média & EP & Média & EP & Média & EP & \\
\hline Cereais & 7,09 & 0,33 & 7,45 & 0,22 & 8,03 & 0,26 & 8,30 & 0,23 & 0,02 \\
\hline Hortaliças & 3,12 & 0,36 & 3,69 & 0,27 & 4,66 & 0,45 & 4,84 & 0,43 & 0,03 \\
\hline Frutas & 2,01 & 0,41 & 2,24 & 0,32 & 3,30 & 0,42 & 3,31 & 0,71 & 0,10 \\
\hline Leite e derivados & 2,66 & 0,30 & 3,14 & 0,26 & 3,98 & 0,47 & 5,82 & 0,52 & $<0,01$ \\
\hline Carnes e ovos & 8,98 & 0,20 & 8,54 & 0,28 & 9,06 & 0,25 & 8,65 & 0,28 & 0,40 \\
\hline Leguminosas & 6,14 & 0,82 & 6,44 & 0,50 & 4,71 & 0,55 & 3,35 & 0,57 & 0,01 \\
\hline Gordura total & 5,44 & 0,46 & 5,60 & 0,36 & 5,36 & 0,32 & 6,25 & 0,39 & 0,37 \\
\hline Colesterol & 8,06 & 0,45 & 8,70 & 0,28 & 8,39 & 0,28 & 7,07 & 0,77 & 0,18 \\
\hline Sódio & 6,54 & 0,32 & 6,26 & 0,28 & 4,98 & 0,62 & 4,07 & 0,62 & $<0,01$ \\
\hline Variedade & 5,58 & 0,43 & 6,06 & 0,25 & 7,00 & 0,39 & 8,15 & 0,37 & $<0,01$ \\
\hline
\end{tabular}

*Cinco indivíduos sem informação sobre escolaridade do chefe da família; * * em relação à escolaridade de 3 a 4 anos de estudo; Utilização do Teste " $t$ " de Wald.

leite e derivados, e variedade de alimentos na dieta, houve diferenças estatisticamente significantes, segundo a escolaridade do chefe da família, ou seja, o aumento no número de anos de estudo apresentou-se associado ao maior consumo dos alimentos incluídos nestes grupos. A relação foi inversa para o grupo das leguminosas e sódio (Tabela 3).

\section{I S C U S S Ã O}

A média de pontos do IQD foi inferior à média dos dados norte-americanos, para as faixas etárias de 5 a 14 anos $(68,4)$ e 15 a 39 anos $(60,2)^{19}$. Isso também foi observado quando comparada aos dados obtidos por Feskanish et al. ${ }^{10}$, ao analisar a dieta ingerida de 426 adolescentes norte-americanos do sexo feminino, de 11 a 14 anos, e 425 do sexo masculino de mesma idade, em que foi constatada, por meio do Healthy Eating Index (HEI), média de pontuação de 63,5 e 62,2 , respectivamente. Além disso, porcentagem significativa de indivíduos apresentou dieta inadequada (28\%), com resultado similar ao estudo realizado por Pinheiro \& Atalah ${ }^{19}$ no Chile, com adaptação do $\mathrm{HEl}$ à 
realidade local, onde a população adolescente chilena apresentou 29,1\% de inadequação na dieta.

Este estudo constatou baixo consumo de hortaliças e frutas, independentemente do sexo e da escolaridade do chefe da família. Resultados semelhantes foram encontrados por Vieira et al. ${ }^{20}$, que verificaram que os alimentos pertencentes ao grupo das hortaliças e frutas são, freqüentemente, rejeitados pelos adolescentes. Vieira et al. ${ }^{20}$ trabalharam com uma população de 185 adolescentes de ambos os sexos, com idade entre $18 \mathrm{e}$ 19 anos, constatando-se que $79,5 \%$ dos estudantes citaram não consumir pelo menos um alimento do grupo de hortaliças e $25,4 \%$ disseram rejeitar uma ou mais frutas.

Vale ressaltar ainda que a média de pontuação foi baixa para o componente leite e derivados, em ambos os sexos. Esse dado também foi encontrado por Troiano et al. ${ }^{21}$, no National Health and Nutrition Examination Surveys (NHANES III), em que o consumo de leite tem diminuído em favor do aumento da ingestão de bebidas gaseificadas (refrigerantes), fato que compromete a densidade óssea, particularmente entre as adolescentes do sexo feminino.

Os indivíduos do sexo feminino apresentaram pontuação maior do IQD no componente colesterol, ou seja, as meninas consumiram menos desse componente. Resultados semelhantes foram encontrados por Garcia et al. ${ }^{22}$, em que, comparativamente, os meninos mostraram maior ingestão desse nutriente do que as meninas, sendo que, aproximadamente, $50 \%$ da população estudada apresentou consumo elevado de colesterol. Capper et al. ${ }^{23}$ também encontraram ingestão de colesterol acima do recomendado, em pesquisa realizada nos Estados Unidos com 200 adolescentes. Obtiveram média de consumo de colesterol de $468 \mathrm{mg} /$ dia para meninos e $297 \mathrm{mg} /$ dia para as meninas.

Em relação aos anos de escolaridade do chefe da família, esta pesquisa mostrou que o consumo de alguns alimentos está associado ao número de anos de estudo. Cesar et al. ${ }^{24}$, avaliando as condições de vida na população em estudo, observaram que o grau de escolaridade do chefe da família mostrou-se um bom indicador das condições de vida descritas em suas diferentes dimensões, uma vez que a amostra foi calculada para avaliar os estratos socioeconômicos da população em função do percentual de chefes de famílias com nível universitário: menos de 5\%; de $5 \%$ a $25 \%$ e com $25 \%$ ou mais.

Segundo Vieira et al. ${ }^{20}$, a condição financeira é um dos fatores determinantes do estilo da alimentação entre os adolescentes, isto é, o maior poder aquisitivo está relacionado à maior aquisição de certos tipos de alimentos. Nos Estados Unidos, ao analisar os dados da Continuing Survey of Food Intakes by Individuals (CSFIII) dos anos de 1994 a 1996, em relação ao IQD para população com mais de 2 anos de idade, observou-se que a pontuação do Índice foi maior entre indivíduos com melhor renda e escolaridade ${ }^{18}$. Pérez-Escamilla \& Haldeman ${ }^{25}$ observaram que a educação formal estava positivamente associada à qualidade da dieta, medida pelo HEl.

Indivíduos com escolaridade mais elevada tendem a possuir maior conhecimento para aquisição de alimentos, aumentando a variedade da dieta, principalmente a partir do maior consumo de frutas e hortaliças e, conseqüentemente, aumentando a pontuação do IQD. O impacto da escolaridade do chefe da família sobre a capacidade de escolha dos alimentos foi encontrado por Fisberg et al. ${ }^{26}$ em estudo com dados dos municípios de Botucatu e Campinas.

Observa-se aumento das médias de escores de cereais, pães e raízes; hortaliças; frutas; leite e derivados, e variedade de alimentos, conforme aumento da escolaridade do chefe da família. Para o grupo das leguminosas foi encontrada situação inversa, provavelmente, o hábito tradicional brasileiro do arroz e feijão prevaleça na população de menor renda.

Estudos recentes demonstram que fatores como níveis de colesterol sérico, excesso de peso, sedentarismo e dieta inadequada estão relacionados com o desenvolvimento de doenças crônicas não transmissíveis, ou seja, hábitos alimentares 
inadequados na adolescência podem levar ao aparecimento desse quadro na vida adulta 3,4,10,20,22 .

Dessa forma, conhecer a qualidade da dieta dos adolescentes torna-se necessário no planejamento de programas de intervenções nutricionais ligados à prevenção de doenças, na tentativa de melhoria do padrão alimentar dos indivíduos aqui estudados.

\section{O N CLUSÃ O}

Com base nos dados obtidos, conclui-se que a maioria dos adolescentes desta amostra não segue as recomendações dietéticas preconizadas, fato o qual pode comprometer a saúde futura desses indivíduos.

\section{REFERÊ NCIAS}

1. Instituto Brasileiro de Geografia e Estatística. Síntese de indicadores sociais 2003. Estudos \& Pesquisas. Informação demográfica e socioeconômica, número 12. Rio de Janeiro; 2004.

2. Heald FP. Nutrition in adolescence. In: PanAmerican Health of adolescents and youths in the Americas. Scientific Publications 489. Washington (DC): PAHOMHO; 1985. p.51-61.

3. Dietz WH. Childhood weight affects adult morbidity and mortality. J Nutr. 1998; $128(2$ Suppl):411S-4S

4. Fisberg M, Bandeira CRS, Bonilha EA, Halpern G, Hirschbruch M. Hábitos alimentares na adolescência. Ped Mod. 2000; 36:734-3.

5. Mertz W. Foods and nutrients. J Am Diet Assoc. 1984; 84(7):769-70.

6. Kant AK, Schatzkin A, Graubard BI, Schairer C. A prospective study of diet quality and mortality in women. JAMA. 2000; 283(16):2109-15.

7. Kennedy ET, Ohls J, Carlon S, Fleming K. The Healthy Eating Index: design and applications, J Am Diet Assoc. 1995; 95(10):1103-8.

8. Hann CS, Rock CL, King I, Drewnowski A. Validation of Healthy Eating Index with use of plasma biomarkers in a clinical sample of women. Am J Clin Nutr. 2001; 74(4):479-86.

9. Weinstein SJ, Vogt TM, Gerrior SA. Healthy Eating Index scores are associated with blood nutrient concentrations in the Third Health and Nutrition
Examination Survey. J Am Diet Assoc. 2004; 104(4):576-84.

10. Feskanich D, Rockett HRH, Colditz GA. Modifying the Healthy Eating Index to assess diet quality in children and adolescents. J Am Diet Assoc. 2004; 104(4):1375-83.

11. U.S. Department of Agriculture. Agricultural Research Service. USDA National Nutrient Database for Standard Reference, Release 16. [tabela de composição de alimentos] 2003; [cited 2004 Jan 28]. Available from: http://www.nal.usda.gov/fnic/ foodcomp

12. Philippi ST. Tabela de composição química de alimentos: suporte para decisão nutricional. Brasília: ANVISA; 2001.

13. Souci SW, Farchman W, Kraut. Food composition and nutrition tables. 5th ed. Stutgard: Medpharm Scientific Publishers; 1994.

14. Pinheiro ABV, Lacerda EMA, Benzecry EH, Gomes MCS, Costa VM. Tabela para avaliação de consumo alimentar em medidas caseiras. 4. ed. São Paulo: Atheneu; 2000.

15. Fisberg RM, Villar BS, editores. Manual de receitas e medidas caseiras para cálculo de inquéritos alimentares: manual elaborado para auxiliar o processamento de inquéritos alimentares. São Paulo: Signus; 2002.

16. Fisberg RM, Slater B, Barros RR, Lima FD, Carandina L, Barros MBA, et al. Índice de qualidade da dieta: avaliação e aplicabilidade. Rev Nutr. 2004; 17(3): 301-18.

17. Bowman SA, Lino M, Gerrior SA, Basiotis PP. The Healthy Eatins Index: 1994-96. Washington (DC): U.S. Departament of Agrilculture; 1998.

18. Variyam JN, Blaylock J, Smallwood D, Basiostis PP. USDA's Healthy Eating Index and Nutrition Information. Washington (DC): United States Department of Agriculture/Economic Research Service; 1998. Technical Bulletin n¹866.

19. Pinheiro AC, Atalah E. Propuesta de uma metodologia de análisis de la calidad global de la alimentación. Rev Med Chile. 2005; 133(2): 175-82.

20. Vieira VCR, Priore SE, Ribeiro SMR, Franceschini SCC, Almeida LP. Perfil socioeconômico, nutricional e de saúde de adolescentes recém: ingressos em uma universidade pública brasileira. Rev Nutr. 2002; 15(3):273-82.

21. Troiano RP, Briefel RR, Carroll MD, Bialostosky K. Energy and fat intakes of children and adolescents in the United States: data from the National Health and Nutrition Examination Surveys. Am J Clin Nutr. 2000; 72(5 Suppl):1343S-53. 
22. Garcia GCB, Gambardella AMD, Frutuoso MFP. Estado nutricional e consumo alimentar de adolescentes de um centro de juventude da cidade de São Paulo. Rev Nutr. 2003; 16(1):41-50.

23. Capper AL, Ellison RC, Witschi JC. Sources of fat, fatty acids, and cholesterol in the diets of adolescents. J Am Diet Assoc. 1990; 90(10): 1429-31.

24. Cesar CLG. Condições de vida da população estudada. In: Saúde e condições de vida em São Paulo: Inquérito multicêntrico de saúde no Estado de São Paulo (ISA-SP). São Paulo: Annablume; 2005. p.65-78.
25. Pérez-Escamilla R, Haldeman L. Food label use modifies association of income with dietary quality J Nutr. 2002; 132(4):768-72.

26. Fisberg RM, Slater B, Morimoto JM, Bueno MB, Cesar CLG, Carandina L, et al. Hábito alimentar da dieta: qualidade da dieta. In: Saúde e condições de vida em São Paulo: Inquérito multicêntrico de saúde no Estado de São Paulo (ISA-SP). São Paulo: Annablume; 2005. p.81-9.

Recebido em: 22/7/2005

Versão final reapresentada em: 14/7/2006

Aprovado em: 18/8/2006 
